beta-catenin (Wnt). These pathways are known to play a role in normal joint development, but are also involved in inflammation, proliferation and apoptosis. Consistently, we observed that HOTAIR and targeted genes were selectively downregulated by TNF- $\alpha$ suggesting a role of HOTAIR in the joint specific regulation of TNF- $\alpha$ mediated inflammatory arthritis.

Disclosure of Interests: Muriel ELHAI: None declared, Mojca Frank-Bertoncelj None declared, Kerstin Klein: None declared, Oliver Distler Grant/research sup port from: Prof. Distler received research funding from Actelion, Bayer, Boehringe Ingelheim and Mitsubishi Tanabe to investigate potential treatments of scleroderma and its complications, Consultant for: Prof. Distler has/had consultancy relationship within the last 3 years with Actelion, AnaMar, Bayer, Boehringer Ingelheim, ChemomAb, espeRare foundation, Genentech/Roche, GSK, Inventiva, Italfarmaco, iQvia, Lilly, medac, Medlmmune, Mitsubishi Tanabe Pharma, Pharmacyclics, Novartis, Pfizer, Sanofi, Serodapharm and UCB in the area of potential treatments of scleroderma and its complications. In addition, he had/has consultancy relationship within the last 3 years with A. Menarini, Amgen, Abbvie, GSK, Mepha, MSD, Pfizer and UCB in the field of arthritides and related disorders, Caroline Ospelt: None declared

DOI: 10.1136/annrheumdis-2019-eular.4370

\section{OP0291 ACTIVATION OF THE DEACETYLASE SIRTUIN-1 COUNTERACTS THE ACTIVATED AND PROANGIOGENIC PROFILE OF ENDOTHELIAL CELLS IN RHEUMATOID ARTHRITIS AND ALLEVIATES EXPERIMENTAL ARTHRITIS}

Agathe Leblond ${ }^{1}$, Sonia Pezet ${ }^{1}$, Anne Cauvet ${ }^{1}$, Claudine Casas ${ }^{1}$, Julie Pires Da Silva ${ }^{2}$, Roxane Hervé ${ }^{3}$, Luca Semerano ${ }^{3}$, Christophe Lemaire ${ }^{2}$, Yannick Allanore ${ }^{1,4}$, Jérôme Avouac ${ }^{1,4}$. ${ }^{1}$ Paris Descartes University, INSERM U1016, Paris, France; ${ }^{2}$ Université Versailles St-Quentin, UMR-S 1180, ChâtenayMalabry, France; ${ }^{3}$ Paris 13 University, Inserm UMR 1125, Bobigny, France; ${ }^{4}$ Paris Descartes University, Cochin Hospital, Rheumatology, Paris, France

Background: Angiogenesis through the recruitment and activation of endothelial cells (ECs) is a crucial event to promote the development of the hyperplasic proliferative synovium in rheumatoid arthritis (RA).

Objectives: Our aim was to decipher the phenotype of ECs derived from circulating progenitors issued from patients with $R A$.

Methods: Proliferation capacities between RA and control ECs was compared using the $x$ CELLigence ${ }^{\text {TM }}$ RTCA System. rh-TNF $\alpha$-induced EC activation was analyzed by adhesion cell expression, VEGF synthesis and stress fiber formation. Angiogenic properties of ECs were assessed in vitro by tube formation on Matrigel and migration capacities through VEGF stimulation in modified Boyden chambers, and in vivo in a mouse model of tumoral neovascularization. Microarray experiments were then performed on Affymetrix GeneChip ${ }^{\circledR}$ Human Exon 1.0 ST Arrays in ECs issued from 18 RA patients compared to 11 age and sex-matched healthy controls to identify gene candidates relevant to pathological angiogenesis. Expression of identified candidates was assessed by RT-PCR and western blots in ECs and by immunohistochemistry in the synovial tissue of RA patients and controls. Their functional importance was then evaluated in vitro after gene invalidation by siRNA and gene overexpression via adenovirus, and in vivo in the mouse model of methyl-BSA-induced arthritis.

Results: EPC-derived ECs issued from RA patients displayed higher proliferation rate, greater sensitization to TNF $\alpha$, with increased VEGF production, ICAM/ VCAM expression, and more prominent stress fiber formation, as well as enhanced angiogenic capacities, characterized by accelerated tube formation and increased migration capacities through VEGF stimulation, compared co control ECs. The subcutaneous transplantation of murine colon carcinoma (CT-26) cells with RA ECs in CB17-SCID mice markedly amplified tumor growth and intratumoral neovessel density, compared to the transplantation of control ECs.

Supervised microarray analyses identified the $\mathrm{NAD}^{+}$-dependent protein deacetylase sirtuin-1 (SIRT1) as a relevant gene candidate. A strikingly decreased SIRT1 gene/protein expression and enzyme activity was detected in RA ECs. A markedly decreased SIRT1 expression was also observed in RA synovium, and more particularly in blood vessels.

Invalidation of SIRT1 with specific siRNA in control ECs was associated with a proliferative and activated profile upon TNF $\alpha$ stimulation, trough the acetylation of p53 and p65, and with the proangiogenic capacities through the upregulation of the matricellular protein CYR61. Conditional deletion of SIRT1 in ECs trough a Cre-LoxP recombination system increased angiogenesis and worsened signs of arthritis and in experimental models of tumor angiogenesis and methyl-BSAinduced arthritis. Conversely, overexpression of SIRT1 via adenovirus in RA ECs reversed this activated and proangiogenic phenotype, and activation of SIRT1 with resveratrol alleviated signs of experimental methyl-BSA-induced arthritis. Conclusion: SIRT1 expression is reduced in synovial blood vessels of patients with RA. SIRT1 invalidation in control ECs reproduces the proliferative, activated and proangiogenic profile of RA ECs, and these effects were reversed by SIRT1 overexpression in RA ECs. These results support the implication of SIRT1 in RA synovial neoangiogenesis and may have direct therapeutic implications, since targeting angiogenesis, and especially SIRT1, might be used as a complementary therapeutic approach in RA.

Acknowledgement: This work was supported by the French Society of Rheumatology (SFR), Arthritis R\&D and a research grand from Pfizer ("Bourse Passerelle").

Disclosure of Interests: Agathe Leblond: None declared, Sonia Pezet: None declared, Anne Cauvet: None declared, Claudine Casas: None declared, Julie Pires Da Silva: None declared, Roxane Hervé: None declared, Luca Semerano Grant/research support from: pfizer, Speakers bureau: pfizer, roche, msd, bms, Christophe Lemaire: None declared, Yannick Allanore Grant/research support from: Inventiva, F Hoffman La-Roche, Sanofi, BMS, Pfizer, Consultant for: Actelion, Bayer, BMS, Boehringer, Roche, Sanofi, Jérôme Avouac Grant/research support from: research grant from Pfizer

DOI: 10.1136/annrheumdis-2019-eular.4516

\section{\begin{tabular}{|l|l}
\hline OP0292 THE HELPFUL EIGHT: KEY SPLICING MACHINERY \\
\hline
\end{tabular} ELEMENTS IN LEUKOCYTE SUBSETS MAY IMPROVE THE TYPIFICATION OF THE DISEASE IN RHEUMATOID ARTHRITIS PATIENTS}

Alejandro Ibañez-Costa ${ }^{1}$, Carlos Perez-Sanchez ${ }^{1}$, Nuria Barbarroja Puerto ${ }^{1}$, Mercedes del Río-Moreno ${ }^{1,2}$, Sergio Pedraza-Arévalo ${ }^{1,2}$, Alejandra M. PatiñoTrives $^{1}$, María Luque-Tévar ${ }^{1}$, Maria del Carmen Abalos-Aguilera ${ }^{1}$, Pedro Seguí Azpilcueta ${ }^{1}$, Pérez Sánchez Laura ${ }^{1}$, Jerusalem Calvo Gutierrez ${ }^{1}$, Rafaela Ortega Castro ${ }^{1}$, Alejandro Escudero Contreras ${ }^{1}$, Eduardo Collantes Estevez ${ }^{1}$,

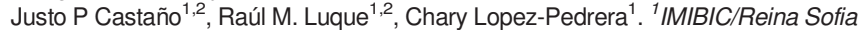
Hospital/University of Cordoba, Cordoba, Spain; ${ }^{2}$ Department of Cell Biology, Physiology and Immunology, University of Cordoba, Cordoba, Spain

Background: The Splicing, a process of removing introns of RNA to produce mature messenger RNA, is exerted by an intricate macromolecular complex, the spliceosome. The alternative splicing, key mechanism that provides transcription and protein diversity, is dysregulated in various genetic, metabolic and inflammatory diseases. Splicing variants could contribute to the development and severity of pathologies of crucial importance.

Objectives: The aim of this study was the identification and characterization of alterations in the splicing machinery of leukocyte subsets of Rheumatoid Arthritis (RA) patients, as well as their influence on the activity of the disease and its atherothrombotic profile.

Methods: Monocytes, lymphocytes and neutrophils from 74 RA patients and 29 healthy donors (HD) were purified, and 45 elements of the splicing machinery were evaluated using a microfluidic qPCR array (Fluidigm). In parallel, the clinical status of the patients and the carotid intima-media thickness (CIMT) were assessed, along with an extensive serological evaluation, including autoantibod ies, oxidative stress markers, inflammatory molecules and prothrombotic mediators. Correlation and association studies and logistic models among those clinica and analytical parameters were performed. Finally, leukocytes from HD were incubated with purified ACPAs-IgG from RA patients and changes promoted in both, splicing machinery and leukocyte inflammatory profile, were assessed.

Results: A significantly altered expression of spliceosome components was found in all the leukocyte subsets: 21,17 and 27 components were differentially expressed in monocytes, lymphocytes and neutrophils, respectively, in RA patient's vs HD. The levels of those altered components were associated to ACPA positivity, high disease activity (DAS28>5.1), early atherosclerosis and radiological involvement. Correlations with inflammatory mediators, oxidative stress markers and NETosis were further identified. Interestingly, eight components, belonging to the major spliceosome (SNRNP70, SNRNP200 and U2AF2), minor spliceosome (RNU4ATAC), and splicing factors (RBM3, RBM17, KHDRS1 and SRSF10) were found simultaneously altered in the three leukocyte subtypes. Logistic regression models, built with levels of simultaneously altered spliceosome components, generated several signatures related to RA disease features that were able to: i) discriminate between RA patients and HD; ii) classify patients with high disease activity (DAS28 $>5.1$ ); iii) recognize patients with radiological involvement; and iv) identify patients showing atheroma plaques. In vitro studies showed that the purified ACPAs of RA patients modulated the expression of these components.

Conclusion: 1) The splicing machinery is profoundly altered in leukocytes from RA patients and closely related to the activity of the disease, including inflammation, autoimmunity and articular involvement, as well as with the occurrence of early atherosclerosis. 2) Evaluation of the common altered components of the spliceosome in leukocytes subsets might be used to typify the disease, avoiding complementary ultrasound or radiological tests.

Acknowledgement: MINECO (FJCl-2016-30825) and ISCIII (PI18/00837 and RIER RD16/0012/0015) co-funded with FEDER.

Disclosure of Interests: None declared

DOI: 10.1136/annrheumdis-2019-eular.7066 